\title{
TIGHT-BINDING MECHANISM IN SLOW LIGHT REGIME
}

\author{
A.E. Akosman ${ }^{1, *}$, M. Mutlu ${ }^{1}$, H. Kurt ${ }^{2}$, and E.Ozbay ${ }^{1}$ \\ ${ }^{1}$ Department of Electrical and Electronics Engineering, Nanotechnology Research Center, Bilkent University, 06800 Ankara, Turkey \\ ${ }^{2}$ Department of Electrical and Electronics Engineering, TOBB University of Economics and Technology, 06560, Ankara, Turkey \\ *akosman@ee.bilkent.edu.tr
}

\begin{abstract}
In this study, tight-binding formalism is applied to a photonic crystal coupled cavity structure in order to investigate the characteristics of ultra slow light modes. Eigen-mode splitting is observed and resulting group indices obtained from the tightbinding formalism and numerical results are compared.
\end{abstract}

Keywords-Photonic Crystals, Photonic Crystal Coupled Cavity Waveguides, Tight Binding Formalism in Electromagnetism

\section{INTRODUCTION}

Slow light phenomenon has attracted significant attention in improving the propagation characteristics of certain electromagnetic (EM) structures. Small group velocity of EM wave is a desired effect in areas such as low threshold lasers [1], some optical communication devices, and buffers [2] and nonlinear optics applications [3]. A photonic crystal (PC) cavity can be formed by introducing a defect to a periodic structure, which provides strong field confinement. In this study, we propose an alternative PC waveguide structure that can be formed by coupling PC cavities. The proposed waveguide has a stronger field confinement compared to a classical PC waveguide, which results in a slower EM field inside the PC. A detailed investigation of this formation has been made in a previous study [4]. In this report, we focus on the theoretical aspect of the ultra slow modes in a PC waveguide constructed by using weakly coupled cavities. The behavior of the ultra slow modes is explained by tight-binding (TB) formalism, which is used to express the nature of the waveguide modes in PCs [5]. TB description in PCs is an analog of classical wave TB explanation, which corresponds to the eigen-mode splitting of the evanescent cavity modes. The impurity states of the semiconductors are an equivalent mechanism to the defect modes of the PC cavity structures. The plane wave expansion (PWM) and the finite different time domain (FDTD) methods are used to calculate the group index values and eigen-mode splitting in the cavity modes. These calculations will be used in extracting TB parameters and in the comparison of TB formalism and numerical results.

\section{THEORETICAL ASPECT OF THE STUDY}

PC waveguides are constructed by introducing a line defect inside the PC structure to control the propagation of the incident EM field with high localization. The frequencies of the allowed modes are in the vicinity of the forbidden band of the $\mathrm{PC}$, which is called photonic band gap (PBG). Furthermore, the modes are bounded by the waveguide mode that is created by the formation of linear defect. It can be deduced that the extra bound is a sign of high confinement and eventually the appearance of slow light modes. The confinement can be increased by introducing dielectric rods of different radii inside the waveguide and coupling cavities. Thus, the bound of the allowed modes is squeezed by the coupled cavity formation, which results in ultra low group velocity. The formation of the PBG inside a classical two dimensional PC waveguide and the alternative coupled cavity structure that is used to investigate the TB formalism in this study is shown in Figure 1.

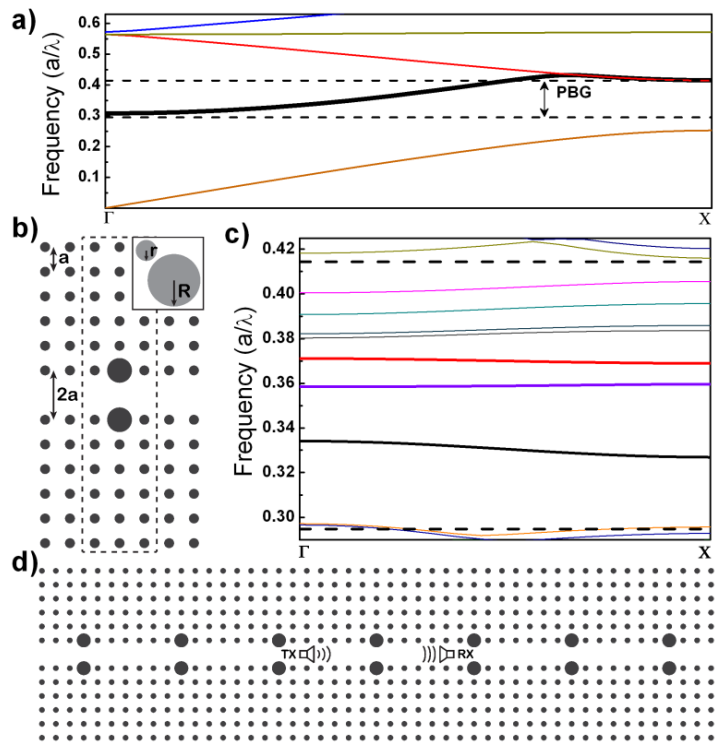

Fig. 1 (a) The dispersion relation under transverse magnetic (TM) field excitation of the two dimensional PC waveguide structure; the black curve is the waveguide mode. (b) The super-cell of the PC coupled cavity configuration. The dashed lines enclose a coupled cavity waveguide, which has smaller coupled cavities (c) The dispersion relation of the PC coupled cavity structure, the studied waveguide bands are given bold, which are observed to be approximately flat bands. (d) The complete PC coupled cavity configuration.

The group velocities of the allowed waveguide modes can be extracted by the dispersion relation. Group velocity of each band can be calculated using the following expression;

$$
v_{g}=d \omega / d k
$$

where $\omega$ represents the angular frequencies of the modes in the frequency range of each band and $k$ is the propagation vector. The refractive index that each mode encounters due to the periodic media can be represented by a new parameter named as group index, which is the analog of the refractive index for dielectric structures. The group index is defined as;

$$
n_{g}=c / v_{g}
$$

where $c$ is the phase velocity of the light in free space. The group index values can be increased to be on the order of $\sim 10^{3}$ [4] by introducing different radius combinations of the 
dielectric rods into the structure. The next step of the study focuses on the demonstration of the compliance of the results obtained by using the TB approach and numerical tools.

\section{REPRESENTATION OF THE TIGHT-BINDING MECHANISM}

The coupled cavities work as an impurity chain in the PC structure. Every cavity that is placed inside the structure creates a new resonance. The resonances eventually converge to a continuous band, creating a waveguide from the cavity chain. Strong coupling between the cavities causes a combined effect, which is crucial to realize the analogy of the TB mechanism.
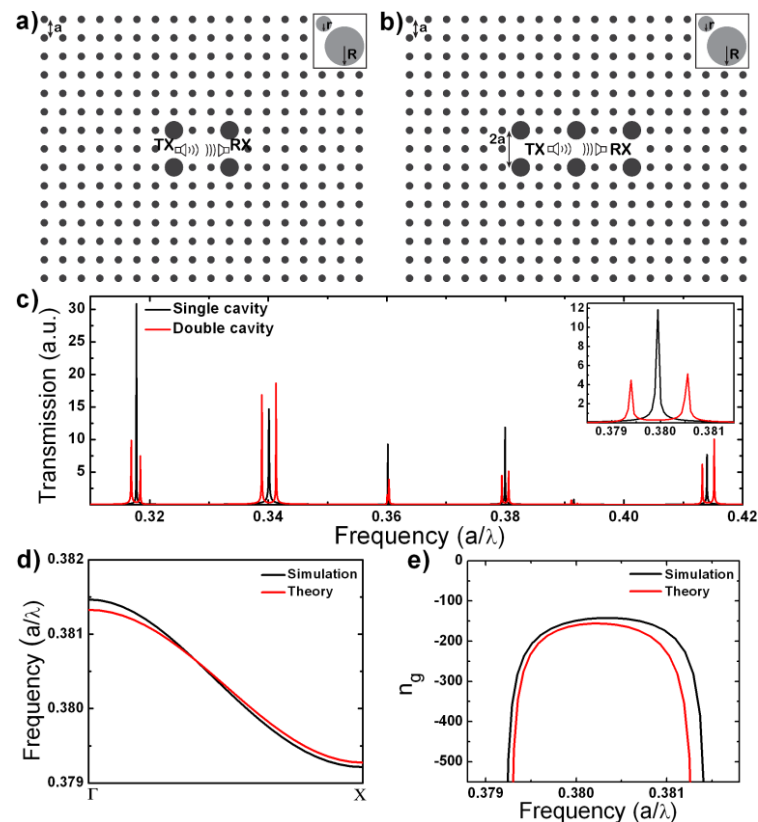

Fig. 2 (a) Single PC cavity formation. (b) Double PC cavity formation to observe eigen-mode splitting. (c) The transmission spectra for the single and double cavity structures. (d) The comparison of dispersion relations of a selected waveguide band of the PC coupled cavity structure, which is constructed by the demonstrated cavities. (e) The comparison of the numerical and theoretical group index values.

Each resonance occurring due to the waveguide can be represented by the eigenmodes of each cavity. The relation between the resonances can be deduced by introducing different number of coupled cavities into the system, which results with an eigen-mode splitting scheme. The eigenmode splitting was expressed as a set of formulation [5]. The eigenmode splitting can be demonstrated by only two different configurations, which are the single cavity and double cavity structures. There is a single resonance peak for the single cavity case centered at $\omega_{c}$. For the double cavity, the two resonance peaks split symmetrically obeying the following relation [5];

$$
\omega_{1,2}=\omega_{c}(1 \pm \beta) /(1 \pm \alpha+\Delta \alpha)
$$

where $\beta, \alpha$ and $\Delta \alpha$ are the TB parameters. $\Delta \alpha$ is very small compared to $\alpha$ and $\beta$, thus it can be ignored. The splitting mechanism is demonstrated using the transmission data of the two configurations as given in Figs. 2 (a) and 2 (b).
The splitting separation decreases by introducing more cavities. The resonance peaks will emerge to each other and form a waveguide band, eventually. The dispersion relation of the occurring waveguide is given as [5];

$$
\omega^{2}(k)=\omega_{c}^{2}(1+2 \beta \cos (k b)) /(1+2 \alpha \cos (k b))
$$

where $b$ is the intercavity distance. The parameters, $\alpha$ and $\beta$ are ignorable compared to unity in flat waveguide modes as in the ultra slow modes. Thus, the dispersion relation can be simplified as [5];

$$
\omega(k)=\omega_{c}(1+\kappa \cos (k b))
$$

where $\kappa$ is equal to $\beta-\alpha$. The group index of a waveguide band can be calculated by combining Eq. 1, Eq. 5 and Eq. 6;

$$
n_{g}=-c /\left(\omega_{c} \kappa b \sin (k b)\right)
$$

The comparison of the tight-binding aspect and the numerical results of a slow light mode are shown in Fig. 2.

Furthermore, the relation between the group index and frequency can be made independent of the constant, $\kappa$, which results in an efficient method to calculate the group index by the knowledge of the transmission spectrum. The relation between $\kappa$ and frequency is given as;

$$
|\kappa|=\Delta \omega /\left(2 \omega_{c}\right)
$$

where $\Delta \omega$ is the frequency bandwidth of the waveguide. Combining Eq. 6 and Eq. 7, the final expression for group index is given as;

$$
n_{g}=-2 c /(\Delta \omega b \sin (k b))
$$

It is apparent in Eq. 8 that group index is inversely related to bandwidth and inter-cavity distance.

\section{CONCLUSIONS}

Tight-binding mechanism is applied to examine the characteristics of the slow light modes of a coupled cavity structure. This formulation provides flexibility in determining the group velocity of an incident field inside the PC by the knowledge of the transmission spectrum, if the waveguide band supports TB mechanism. Furthermore, the characteristics of ultra slow light modes can easily be determined without phase measurements. The experimental verification of TB formalism will be performed as a near-future work.

\section{REFERENCES}

[1] M. Loncar, T. Yoshie, A. Scherer, P. Gogna and Y.Qiu, "Low-threshold photonic crystal laser", Appl. Phys. Lett., vol. 81, pp. 2680-2682, 2002.

[2] M. L. Povinelli, S. G. Johnson and J.D. Joannopoulos, "Slow-light band edge waveguides for tunable time delays.”, Opt. Express, vol. 13, pp. 7145-7159, 2005.

[3] M. Soljacic and J.D. Joannopoulos, "Enhancement of nonlinear effects using photonic crystals. ”, Nat. Mater., vol. 3, pp. 211-219, 2004.

[4] K. Ustun and H. Kurt, "Ultra slow light achievement in photonic crystals by merging coupled cavities with waveguides", Opt. Express, vol. 18, pp. 21155-21161, 2010

[5] M. Bayindir, B. Temelkuran, E. Ozbay, "Tight-Binding description of the coupled defect modes in three-dimensional photonic crystals", Phys. Rev. Lett., vol. 84, pp. 2140-2143, 2000. 